been rather scanty, and so any conclusions that may be formed must be taken with reserve. I scarcely think that there is any direct relationship between gout per se and the retention of the pressor bases, and yet in most cases which I have examined the diminution in the excretion of these bases is not proportional to the increase of arterial pressure observed; for example, the patient with a systolic pressure of $135 \mathrm{~mm} . \mathrm{Hg}$ excreted about half the usual quantity found in normal urine, as judged by the intensity of the physiological effect.

6. In cases where the urine contains a deposit of uric acid there is a marked diminution of the colloid matter of that fluid. This colloid consists of or contains a sulphur compound-chondroitin sulphuric acid-which is a constituent of cartilage. The association of this condition with the frequency of urate deposition in cartilage is suggestive, and deserves fuller investigation.

I have great pleasure in acknowledging my indebtedness to Professor W. D. Halliburton for the use of his laboratory during my work and for his help in the blood pressure experiments, and to Dr. Rosenheim for his courtesy and suggestive aid during the investigation. To Dr. Mott and Dr. J. C. Muir I tender my cordial thanks for the specimens of urine which they have sent to me.

Harrogate.

\section{THE OBSTRUCTION OF THE ILEUM WHICH DEVELOPS IN CHRONIC INTESTINAL STASIS.}

BY W. ARBUTHNOT LANE, M.S. LOND., H.R.C.S. ENG., SURGEON TO GUY'S HOSPITAL AND SENIOR SURGEON TO THE HOSPITAI FOK SICK CHILDREN, GREAT ORMOND-STREET.

IN describing the causation, pathology, and treatment of chronic intestinal stasis $I$ have illustrated and explained the changes in the appendix and ileum which arise in consequence of the progressive descent of the cæcum into the pelvis which takes place in this condition. In this communication I wish if possible to accentuate especially the change in the ileum in order to call attention to it in a more definite manner. I do so because it is most important and far-reaching in its influence for harm, and it has apparently not attracted the attention of any of the readers of my papers.

The cæcum loaded up with its fluid contents tends in the erect posture of the trunk to glide downwards and inwards into the true pelvis, which it struggles to occupy, together with the transverse colon and sigmoid, not only to the disadvantage of these structures themselves but also to the distinct detriment of the organs which exist normally in it. The mere hypertrophy of the muscular wall of this portion of the large bowel is insufficient to meet the excessive fluid pressure exerted upon it, and it gradually dilates and descends from its normal position. This tendency to falling of the distended cæcum is met by the formation of two series of resistances which develop as independent bands or thickenings in peritoneum. The thickening in the peritoneum is the first step from which the separate band or mesentery is subsequently developed.

The external series of peritoneal adhesions, bands, or mesenteries extends from the outer aspect of the cæcum upwards and outwards to the peritoneum lining the abdominal wall external to it (see Figure). The lowest of these not infrequently attaches the appendix, securing it in the first instance at its base and extending a variable distance along its length. In this manner the fixed portion of the appendix forms part of one of the ligaments or ties opposing the downward displacement of the cæcum. The result of strain exerted upon the appendix when the cæcum is full is to kink the distal mobile portion of the appendix upon that part which has taken on the function of a ligament and to partly or completely obstruct the lumen of its mucous lining. Such obstruction produces an accumulation in the distal portion of a transient or permanent character, and a condition which is described generally as appendicitis. In some cases a mass or concretion develops from the accumulated inspissated contents.

The other limb of the parallelogram of forces lies internal to the cæcum, and is an added strain along that portion of the mesentery which holds up the end of the ileum. consequence of this strain a thickening forms on the under surface of the mesentery, and this contracts the mesentery along the line of strain. This exerts a drag upon the ileum at a point about two inches from its termination. In this way that piece of the ileum external to the area of thickening in the mesentery, together with that same thickening, form an internal resistance which holds up the dilated cæcum heavy with fluid contents and opposes its downward displacement. Therefore the last two inches of the ileum beyond the new band performs in addition to its normal function that of an internal ligament of the crecum, precisely in the same way as the fixed portion of the appendix with the band securing

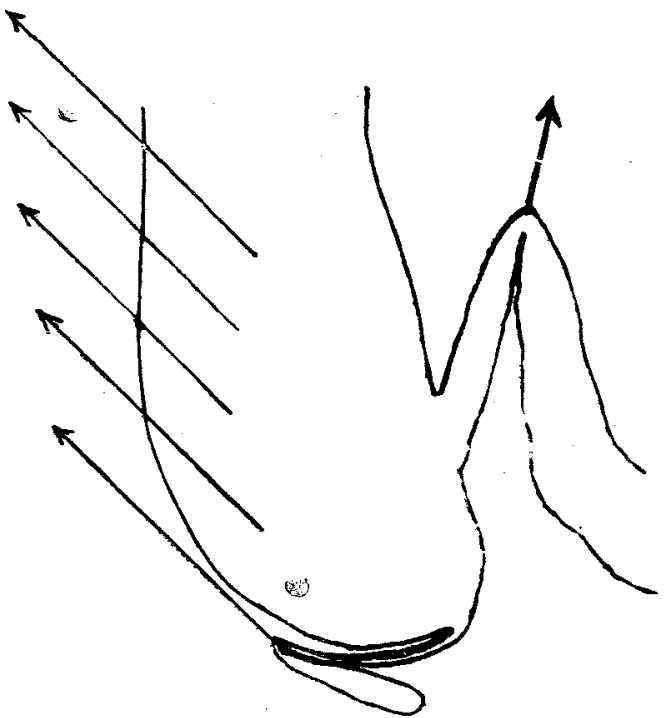

Diagram indicating peritoneal adhesions opposing the downward displacement of the loaded cæcum. The last adhesion is attached to the appendix.

it forms an external ligament of the creum. Again, as in the case of the appendix, the strain exerted upon the fixed portion of the ileum serves to reduce the calibre of its lumen and to produce an obstruction to the passage of fæcal matter through which it is damming back the material in the small intestine. This fæcal accumulation remaining for an $a b$. normally long period itself undergoes changes and produces alterations in the irtestines, which are experienced as discomfort, pain, or distress to the patient.

Clinically a varying degree of impairment of the function of the appendix is frequently associated with a similar defect in the end of the ileum. It is by no means necessary that the conditions should be associated, since the appendix may escape the grip of these newly formed peritoneal ties even in advanced cases of intestinal stasis. In proportion, however, as the appendix escapes performing its share in restraining the cacum from getting too far into the pelvis, the greater is the proportion of strain thrown on the affected mesentery of the ileum and the more likely is this tube to be constricted by thickenings or bands. As indicated before, all these ties are represented in the first instances as thickened lines on the peritoneum and later as mesenteries free from the peritoneum to which they were originally attached. The skeleton represents a crystallisation of lines of force which when transmitted in one direction are laid down as compact tissue and when in varying directions as cancellous tissue. In precisely the same way the peritoneal thickenings and the bands or mesenteries into which they develop later represent the crystallisation of lines of strain and are evolutionary and functional, and have no relationship whatever with any inflammatory process.

The symptoms produced by the kink in the ileum are quite distinct from those which result from trouble in the appendix. They may be, and often are, associated, being produced by the same cause. The tenderness of the kink in the ileum extends from the umbilicus almost directly downwards and to the right, and is quite separate from the pain and tenderness of a fixed appendix. The distension of the small intestine is a marked feature and varies greatly with the time of day, since it is much worse when the abdominal wall is lax and tired. The pain develops or becomes accentuated at a more or less regular interval after the ingestion of food. It is increased by any jerky movement, as riding, running, especially up or down stairs, \&c.

This kinking of the ileum is the cause of much of the pain 
and discomfort that remains after the removal of the appendix. If the appendix has also been in trouble the pain and tenderness produced by it will have been removed by the operation, but the pain consequent on the kinking of the ileum still remains and is the cause of much disappointment both to the surgeon as well as to the patient. I would call the attention of the profession to the importance of this condition.

Cavendish-square, W.

\section{SIGMOIDITIS AND MESO-SIGMOIDITIS.}

BY R. P. ROWLANDS, M.S. LoND., F.R.C.S. ENG., ASSISTANT SURGEON, GUY'S HOSPITAL, LONDON.

DURING the last 18 months I have met with two interesting examples of this comparatively rare condition. They seem to me to be of sufficient interest and importance to merit publication, especially as the disease, particularly in this country, has not received the attention it deserves, with the result that it is often mistaken for malignant disease. The clinical symptoms and physical signs may closely simulate those of carcinoma of the sigmoid colon. It is very difficult to distinguish the two conditions even when the abdomen is opened. In at least 12 of the 25 instances of stenosis collected by Telling, a diagnosis of carcinoma was made either before or at operation. The true nature of the disease may be realised only after resection or even after microscopic examination. When the extent of the "growth" or the condition of the patient in the presence of acute obstruction does not allow a radical operation, the mistake may not be discovered. The unusual prolongation of life after colostomy or the spontaneous disappearance of the "growth" may give an indication of the true state of things. Most of us know of several instances of this kind. The writer knows of at least three patients who have survived for many years after colostomy for supposed carcinoma of the pelvic colon. Dr. W. H. Maxwell Telling in his able paper upon Acquired Diverticula of the Sigmoid Flexure ${ }^{1}$ has pointed out that several museum specimens long regarded as examples of carcinoma of the sigmoid are really inflammatory. He draws special attention to one of the most important causes of simple inflammation of the sigmoid flexure, "the presence of acquired diverticula in or near the sigmoid colon."

It is very easy to overlook these diverticula, especially when they are limited to the mesenteric border. They project into a greatly thickened mesentery, and their orifices are often so small that they can only be discovered by means of a probe. They are so hidden that they can be very easily overlooked. The writer would certainly have overlooked them if he had not previously read Dr. Telling's paper. They are especially common at the mesenteric border follow. ing the blood-vessels, where the muscular wall of the bowel is often deficient and ill supported. The retention of irritating and infective material in the diverticula may lead to inflammation and even to perforation of the thin-walled sacs. This may be followed by spreading or lncalised peritonitis, but when the diverticula are deeply emberded in a thick mesentery the inflammation may be confined to the mesentery and the outer walls of the bowel. In some cases suppuration may take place between the layers of the mesentery, and pus may discharge into the bowel or into a diverticulum. In some it may burst into the peritoneal cavity. In many cases appendicitis may be closely simulated except that the swelling is on the wrong side. Sometimes the inflammation is more chronic and leads to much thickening and shortening of the mesentery and interstitial inflammation of the intestinal wall with secondary stenosis. The mucous membrane may remain fairly healthy for a long time. The condition is by no means limited to the sigmoid colon. Thus in one of my patients it extended from the pelvic colon to the splenic flexure. In others it has been limited to the splenic flexure or to a short segment of the transverse colon. The formation of diverticula in the colon seems to be chiefly due to longcontinued constipation associated with failure of the muscularity of the intestinal wall. It is therefore a condition which is not of ten seen before middle age, and Telling points out that "the average age of those in which the

1 The Lancet, March 21st (p. 843) and 28th (p. 928), 1908 diverticula caused clinical symptoms was 55 years" (47 cases). It is, of course, admitted that the presence of diverticula is not the only cause of inflammatory stenosis of the large intestine. Tuberculous infiltration, especially of the cæcum and ascending colon, may closely simulate malignant disease. Gonorrhceal, syphilitic, and tuberculous tubular stenosis of the rectum are well-known conditions. The spread of inflammation from the female pelvic organs may give rise to true stenosis of the sigmoid or pelvic colon.

Detailed accounts of my two cases may now be given. They are of especial interest for the two following reasons : 1 . Both of them were mistaken by me and others before exploration for growth of the descending or sigmoid colon. It is possible that the mistake would not have been made if sigmoiditis had been borne in mind. In any case I do not think that a correct diagnosis would have materially altered the treatment, but the prognosis given before the operation would have been different. 2. Circumstances made it necessary for different operations to be performed for the two patients. In one the ileum was short-circuited to the front wall of the pelvic colon 5 inches from the anus. This saved the patient's life and made her much more comfortable. It improved her general condition, but it has not cured her. In the other the diseased part was resected with complete success.

CASE 1. - The first case was that of a lady, aged 60 years, a patient of Dr. J. Kidd. She had suffered from constipation for many years. There were only two actions of the bowels in a fortnight during May, 1908, while the patient was on a holiday at Lugano. The symptoms had got very much worse by July 21st, 1908, when she was seized with severe colicky pains in the abdomen. The bowels were opened with difficulty, so that opening medicines had to be taken regularly, and these were followed by liquid motions. During the last few weeks liquid stools and slime were frequent, but no formed motions were ever passed. Tenesmus had been very severe and spurious diarrhoea urgent and often uncontrollable, so that a diaper had to be worn. Usually only mucus and flatus escaped, but this gave great relief. Flatulence had been very troublesome. The wasting had been considerable. On examination the patient was found to be thin, shrivelled and anæmic. The bowels had not been opened for three days. The tongue was furred and white, and the abdomen was distended but quite soft and supple. Obvious peristalsis could be seen, and this was especially marked in the transverse colon. The descending and sigmoid colon could be felt in the iliac fossa, and the swelling and hardness extended up into the left loin and under the cover of the ribs near the splenic flexure, where the patient said that she had had much pain and had noticed gurgling. There was shifting dulness in the flanks. The transverse colon was greatly dilated. There was no tumour felt on pelvic examination. A systolic apical bruit was heard and the ankles were cedematous. Chronic intestinal obstruction dne to growth of the descending colon was diagnosed, and an operation advised for its relief. The patient expressed strong disapproval of colostomy, but this did not seem likely to be necessary, because either resection or lateral anastomosis was thought to be possible. Upon npening the abdomen on August 22 nd about two pints of sanious fluid escaped. The colon from just below the sacro-iliac synchondrosis to the splenic flexure was greatly thickened, red, and codematous. It formed a rigid tube with a very thick wall and very narrow lumen. The mesentery had shortened so much that only a very short meso-sigmoid could be found, and there was no sigmoid loop of bowel, but only a nearly straight rigid tube, ending abruptly at the splenic flexure. There was no meso-colon. The peritoneal tissues on either side and behind the colon were cedematous. In view of the patient's feeble condition and the presence of subacute obstruction which was nearly complete, and the condition of the distended transverse colon which was full of hard scybala, immediate resection was considered inadrisable and too risky. Moreover, the extent of bowel to be removed was very considerable, and the patient had refused her permission to a colostomy. It was therefore decided to join the ileum to the front of the pelvic colon or rectum at the lower border of the disease. Even there the bowel was not quite healthy, and it was so fixed that the anastomosis was very difficult. The friable condition of the large intestine and the reed to make the opening very low down added to the difficulties. However, with the aid of clamps and the Trendelenburg 